J. Dairy Sci. 92:87-94

doi:10.3168/jds.2008-1449

(c) American Dairy Science Association, 2009.

\title{
Cheddar cheese classification based on flavor quality using a novel extraction method and Fourier transform infrared spectroscopy
}

\author{
A. Subramanian, W. J. Harper, and L. E. Rodriguez-Saona ${ }^{1}$ \\ Department of Food Science and Technology, The Ohio State University, 2015 Fyffe Court, Columbus 43210
}

\section{ABSTRACT}

Analysis of Cheddar cheese flavor using trained sensory and grading panels is expensive and time consuming. A rapid and simple solvent extraction procedure in combination with Fourier transform infrared spectroscopy was developed for classifying Cheddar cheese based on flavor quality. Fifteen Cheddar cheese samples from 2 commercial production plants were ground into powders using liquid nitrogen. The watersoluble compounds from the cheese powder, without interfering compounds such as fat and protein, were extracted using water, chloroform, and ethanol. Aliquots $(10 \mu \mathrm{L})$ of the extract were placed on a zinc selenide crystal, vacuum dried, and scanned in the mid-infrared region $\left(4,000\right.$ to $\left.700 \mathrm{~cm}^{-1}\right)$. The infrared spectra were analyzed by soft independent modeling of class analogy (SIMCA) for pattern recognition. Sensory flavor quality of these cheeses was determined by trained quality assurance personnel in the production facilities. The SIMCA models provided 3-dimensional classification plots in which all the 15 cheese samples formed wellseparated clusters. The orientation of the clusters in 3-dimensional space correlated well with their cheese flavor characteristics (fermented, unclean, low flavor, sour, good Cheddar, and so on). The discrimination of the samples in the SIMCA plot was mainly due to organic acids, fatty acids and their esters, and amino acids (1,450 to 1,350 and 1,200 to $990 \mathrm{~cm}^{-1}$ ), which are known to contribute significantly to cheese flavor. The total analysis time, including the sample preparation time, was less than $20 \mathrm{~min}$ per sample. This technique can be a rapid, inexpensive, and simple tool to the cheese industry for predicting the flavor quality of cheese.

Key words: Cheddar cheese, flavor quality, infrared spectroscopy

Received June 11, 2008.

Accepted September 14, 2008.

${ }^{1}$ Corresponding author: rodriguez-saona.1@osu.edu

\section{INTRODUCTION}

About 9.13 billion pounds of cheese was produced in the United States in 2005, and around 33\% of the total production was Cheddar cheese (NASS, 2007). One of the most important parameters that determines the marketability of the cheese is its flavor. Undesirable flavors can reduce the acceptance and the price of the cheese significantly. The characteristic flavor, aroma, texture, and appearance of cheese develop during ripening. Cheese ripening or maturation is a slow process $(2$ to $24 \mathrm{mo}$ ) characterized by a series of complex physical, chemical, and microbiological changes affecting the principal components of the cheese (Singh et al., 2003). The complex nature of the cheese ripening process makes it a challenge to produce cheese of uniform sensory properties, especially with respect to flavor.

The flavor profiles of cheese are complex and variety- or type-specific (Akalin et al., 2002) and are determined to a great extent by proteolysis, lipolysis, and glycolysis during ripening (Bachmann et al., 1999; Singh et al., 2003). The effect of the manufacturing process, the composition of the milk (such as protein and fat levels), and the biochemical events that occur during ripening will influence the final quality of the cheese (Chen et al., 1998). The principal compounds that contribute to the flavor include organic acids, amino acids, sulfur compounds, lactones, methyl ketones, alcohols, and phenolic substances (Seitz, 1990; Urbach, 1993). Currently, cheese flavor and grades are determined by trained panels. This approach is complex, expensive, and time consuming. Furthermore, it is practically difficult to taste and evaluate each batch of cheese. Hence, there is a need for rapid and reliable instrumental methods to determine the flavor quality of cheese. A rapid method, aside from saving time and money for the cheese industry, will help in ensuring better product quality.

The "component balance theory" put forth by Kosikowski and Mocquot (1958) suggests that Cheddar cheese flavor is produced by the correct balance and concentration of a range of sapid and aromatic compounds. Hundreds of compounds have been identified that contribute to cheese flavor (McSweeney and Sousa, 
2000). Hence, an instrumental method that is capable of simultaneously monitoring multiple compounds and their functional groups is necessary. Furthermore, cheese composition is very complex with many interfering components. Sample heterogeneity is also very common. Attempts have been directed at finding and evaluating microbiological and biochemical parameters by which cheese could be classified and whereby uniform cheese quality could be established (Adda et al., 1982; Farkye and Fox, 1990; Seitz, 1990; Singh et al., 2003). Methods such as HPLC (Akalin et al., 2002; Lues and Bekker, 2002), GC (Partidario et al., 1998; Thierry et al., 1999), and MS (Alli et al., 1998) have been investigated for analysis of cheese components. These studies have made significant contributions to the understanding of the ripening process. However, the methods are complicated and time consuming; require different conditions and accessories for analyzing different classes of compounds; and have limited applications as routine quality control methods for overall cheese flavor quality. Because of the above reasons, no reliable instrumental methods exist for rapid analysis of flavor quality.

Fourier transform infrared (FT-IR) spectroscopy is a simple, rapid, and reliable technique that has been widely researched and applied for analysis of food components. It is based on the principle that different chemical functional groups require different amounts of energy (different wavelengths) for excitation. Fourier transform infrared spectroscopy $\left(4,000\right.$ to $\left.700 \mathrm{~cm}^{-1}\right)$ monitors the absorbance of infrared light by functional groups in the sample to provide a chemical fingerprint (spectrum) of the sample that shows the overall chemical composition of the sample. Fourier transform infrared spectroscopy combined with multivariate analysis has been suggested for rapid analysis of cheese by many researchers. Examples of its applications in cheese analysis include determination of fat, moisture, and protein analysis (McQueen et al., 1995; Chen et al., 1998; Chen and Irudayaraj, 1998), shelf-life analysis of Crescenza cheese (Cattaneo et al., 2005), compositional analysis of Swiss cheese (Rodriguez-Saona et al., 2006), and determination of geographic origin of cheese (Karoui et al., 2004).

Almost all studies on analysis of cheese by FT-IR have been directed toward monitoring major food components such as protein and fat. Limited research has been conducted on the use of FT-IR spectroscopy for analysis of minor components that contribute to the flavor. This is mainly because of difficulties in the sampling procedures and the heterogeneous nature of cheese (McQueen et al., 1995). Koca et al. (2007) reported a correlation coefficient of $>0.90$ between acetic, propionic, and butyric acids contents and the FT-IR spectra. Because of interferences from fat and protein, a complex water-soluble extraction method was followed to prepare the samples for FT-IR analysis. The objective of this research was to develop a simple sample preparation procedure that will provide water-soluble extracts that have well-defined and consistent spectra, which when analyzed through multivariate statistical classification techniques could rapidly classify Cheddar cheese based on flavor quality. To the best of our knowledge this is the first research on rapid flavor quality analysis of cheese using FT-IR spectroscopy.

\section{MATERIALS AND METHODS}

\section{Cheddar Cheese Samples}

Fifteen different Cheddar cheese samples, ripened for $70 \mathrm{~d}$, were obtained from 2 production plants of a commercial cheese manufacturer. Five cheese samples were obtained from a commercial cheese manufacturing facility (plant 1) and 10 samples were obtained from another production plant (plant 2). The sensory flavor quality of these cheeses was earlier analyzed by trained quality assurance personnel in the production facilities and provided along with the cheese samples. Cheese samples were vacuum-packaged and stored at $-40^{\circ} \mathrm{C}$ until analysis.

\section{Extraction of Flavor Compounds for FT-IR Analysis}

A novel extraction procedure using water, chloroform, and ethanol was used to prepare the samples (Subramanian and Rodriguez-Saona, 2008). About 20 $\mathrm{g}$ of frozen cheese sample was cut into small pieces. The pieces were mixed with about $20 \mathrm{~mL}$ of liquid nitrogen to freeze them rapidly. The hard-frozen pieces were cryogenically ground in a blender and the powdered cheese was then kept frozen $\left(-40^{\circ} \mathrm{C}\right)$ until extraction. For extraction, exactly $0.1 \mathrm{~g}$ of the powdered cheese was weighed and $0.5 \mathrm{~mL}$ of distilled water was added and mixed well. The mixture was sonicated using an ultrasonic dismembrator (Fisher Scientific, Pittsburgh, $\mathrm{PA}$ ) for $10 \mathrm{~s}$ to break down clumps and improve the extraction of water-soluble components from cheese powder. An equal amount $(0.5 \mathrm{~mL})$ of chloroform was added to the cheese powder-water mixture (to separate complex fat), mixed well, and centrifuged at 15,700 $\times$ $g$ and $25^{\circ} \mathrm{C}$ for $3 \mathrm{~min}$. Exactly $200 \mu \mathrm{L}$ of the resulting supernatant was mixed with an equal amount of $100 \%$ ethanol (to precipitate complex proteins), and centrifuged at $15,700 \times g$ and $25^{\circ} \mathrm{C}$ for $3 \mathrm{~min}$. Supernatant $(100 \mu \mathrm{L})$ was used for FT-IR analysis. 


\section{FT-IR Spectroscopy}

Fourier transform infrared spectroscopy of the cheese samples was carried out on a Varian 3100 FT-IR spectrometer (Varian Inc., Palo Alto, CA) equipped with a Permaglow mid-infrared source, extended-range potassium bromate infrared beam splitter, and deuterated triglycine sulfate detector. Aliquots $(10 \mu \mathrm{L})$ of the extracts were placed on a 3-bounce MIRacle attenuated total reflectance accessory with an zinc selenide crystal (Pike Technologies, Madison, WI) and vacuum dried to form a thin film. Infrared spectra were recorded between 4,000 and $700 \mathrm{~cm}^{-1}$ at a resolution of $8 \mathrm{~cm}^{-1}$, using the data collection software (Varian Resolutions Pro v4.05, Varian Inc.). To improve the signal to noise ratio, 128 scans were averaged for each spectrum. For each cheese, 3 samples were collected from different locations and powdered. Five independent extractions were made for each powder and 3 spectra were collected per extract. Hence, for the 5 samples from plant 1, a total of 225
( 5 cheeses $\times 3$ powders per cheese $\times 5$ extractions per powder $\times 3$ spectra per extract) spectra were collected. For the 10 samples from plant 2, a total of 450 spectra were collected.

\section{Multivariate Analysis}

Multivariate analyses of the data were carried out using a commercially available comprehensive chemometrics modeling software called Pirouette (v3.11, Infometrix Inc., Woodville, WA). For analysis, spectra were imported into Pirouette, mean-centered, transformed into their second derivative using a Savitzky-Golay polynomial filter (5-point window), and vector-length normalized. For classifying the cheese samples based on their sensory flavor quality, the spectra were analyzed by soft independent modeling of class analogy (SIMCA); SIMCA is a classification algorithm based on principal component (PC) analysis. Based on specific fingerprint spectral information from infrared light absorbance

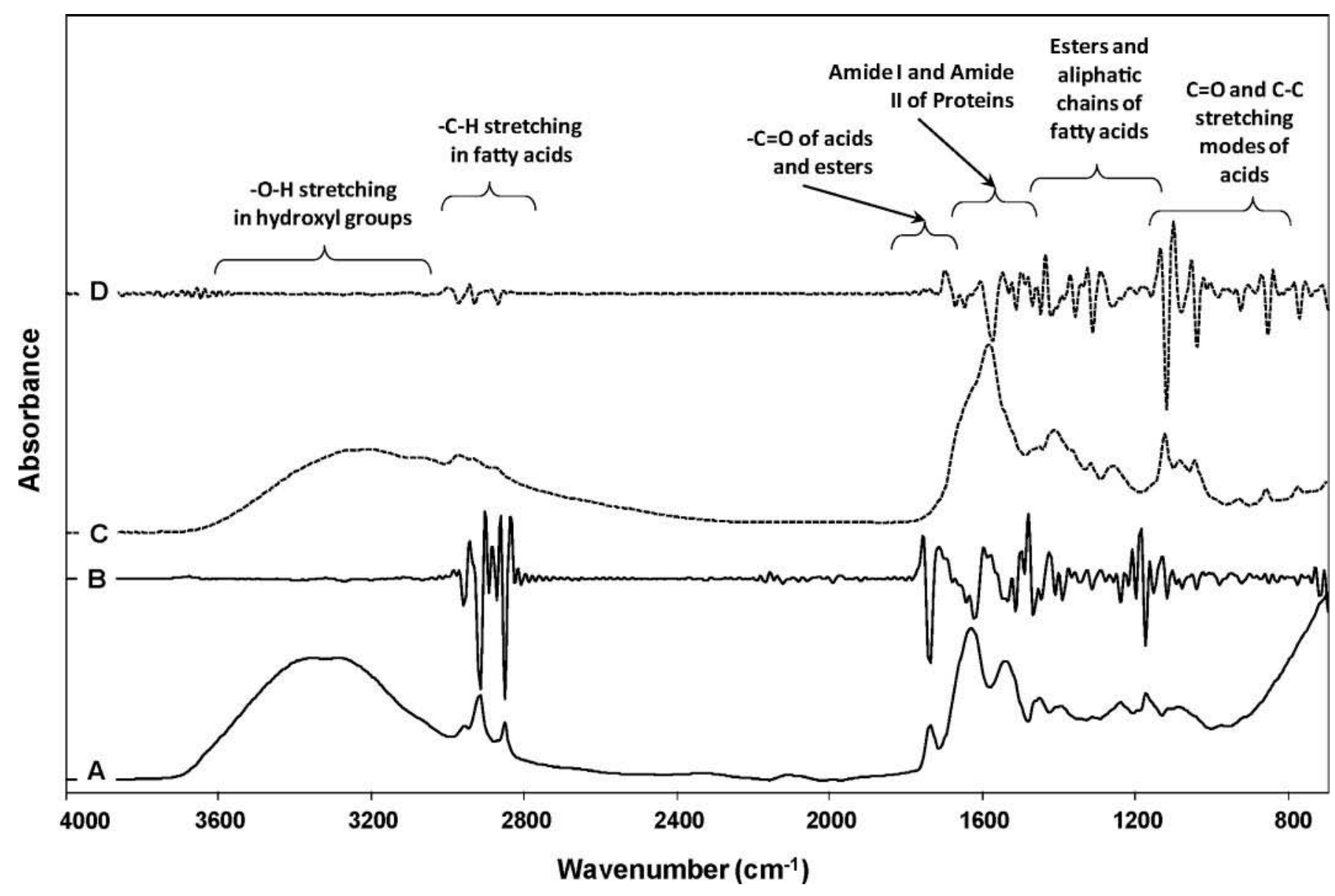

Figure 1. Raw (A and C) and derivatized (B and D) spectra of Cheddar cheese (- - ) and Cheddar cheese extract (---). Certain important functional groups and their region of absorbance are highlighted. Spectral data were obtained in the mid-infrared region $\left(4,000\right.$ to $\left.700 \mathrm{~cm}^{-1}\right)$ at resolution of $8 \mathrm{~cm}^{-1}$ by co-adding 128 scans. Cheddar cheese spectra were obtained by pressing $0.5 \mathrm{~g}$ of cheese on a diamond attenuated total reflectance (ATR) crystal. Extracts were scanned by drying $10 \mu \mathrm{L}$ of the extract on a zinc selenide ATR crystal. 
by functional groups in cheeses, multivariate analysis modeled the relationships between large numbers of dependant variables to classify different cheeses. The data were projected onto the first $3 \mathrm{PC}$ axes to visualize clustering of samples in 3-dimensional (3D) space based on their flavor quality (fermented, sour, good cheddar, and so on). The spectral regions influencing the classification of the cheeses were determined from the measure of variable importance (discriminating power).

\section{RESULTS AND DISCUSSION}

\section{FT-IR Spectra of Cheddar Cheese}

The FT-IR spectra of Cheddar cheese samples were collected in the mid-infrared region $\left(4,000\right.$ to $\left.700 \mathrm{~cm}^{-1}\right)$, using a 3-bounce attenuated total reflectance crystal. In a 3-bounce crystal, infrared light bounces on the sample 3 times, increasing the absorbance and hence the signal. For analysis, the raw spectra were normalized, standardized, and transformed to their second derivatives to remove the baseline shifts, improve the peak resolution, and reduce the variability between replicates (Kansiz et al., 1999). A typical mid-infrared spectrum of Cheddar cheese and its second derivative are shown by lines A and B, respectively, in Figure 1. The spectra of Cheddar cheese were marked by absorbance from complex fat and protein in the regions 3,000 to 2,800 and 1,800 to $1,000 \mathrm{~cm}^{-1}$, respectively, which were very similar to observations reported by several other researchers (Chen et al., 1998; Chen and Irudayaraj, 1998; Rodriguez-Saona et al., 2006; Koca et al., 2007). Asymmetric and symmetric stretching vibrations of $\mathrm{C}-\mathrm{H}$ groups in methylene groups of longchain fatty acids were observed in the region 3,000 to $2,800 \mathrm{~cm}^{-1}$. Strong signal from the $\mathrm{C}=\mathrm{O}$ groups of fatty acid esters were also present at $1,740 \mathrm{~cm}^{-1}$. Broad amide I and amide II bands of proteins peaked at around 1,640 and $1,540 \mathrm{~cm}^{-1}$, respectively. The spectra also included several other bands in the region 1,800 to $900 \mathrm{~cm}^{-1}$, primarily due to $\mathrm{C}-\mathrm{H}$ bending, C$\mathrm{O}-\mathrm{H}$ in-plane bending, and $\mathrm{C}-\mathrm{O}$ stretching vibrations of lipids, organic acids, amino acids, and carbohydrate derivatives that play a significant role in cheese flavor. The region from 1,800 to $900 \mathrm{~cm}^{-1}$ exhibited poor peak definition because of multiple absorptions and signal masking by major compounds. To analyze Cheddar cheese flavor, interferences and variability caused by macromolecules such as protein and fat have to be minimized and peak definition has to be improved. This emphasized the need for an extraction method to improve spectral information.

\section{FT-IR Spectra of Water-Soluble Fractions}

Water-soluble fractions of Cheddar cheese have been reported to contribute significantly more to Cheddar cheese flavor intensity than complex fat, fat-soluble volatiles, or insoluble fractions by several researchers (Aston and Creamer, 1986; Engel et al., 1997, 2002). Several different extraction procedures with various solvents were investigated to obtain consistent spectra that would enable classification of the cheese based on flavor. Extraction using water, chloroform, and ethanol was found to provide extracts of consistent composition that enabled effective discrimination of Cheddar cheese based on flavor. Drying of the extract on the crystal resulted in the formation of a uniform film of sample. The presence of ethanol in the extract helped in faster drying of the samples on the crystal. The drying time per sample was $3 \mathrm{~min}$. This sample preparation method allowed for the collection of high-quality spectra with distinct spectral features that were very consistent within each sample.

Raw and derivatized FT-IR spectra of the Cheddar cheese extract are shown in Figure 1 (lines C and D). Complex fat and protein that play a limited role in the flavor of Cheddar cheese were removed by sequential extraction with chloroform and ethanol. Chloroform extracted the long-chain fatty acids from the cheese powder-water mixture. This was confirmed by the signal reduction in the region 3,000 to 2,800 and $1,740 \mathrm{~cm}^{-1}$ in the spectra of cheese extract (Figure 1). Ethanol, with its high affinity to water, removed water from the protein, causing proteins to form complexes and precipitate on centrifugation. As evident from Figure 1, the absorption from amide I and amide II bands of proteins reduced significantly in the spectra of cheese extract. Additionally, ethanol helped in extracting compounds with slight nonpolarity, including short- and mediumchain fatty acids, from the cheese. The resulting extract contained water-soluble components from the cheese, which included organic acids, short- and medium-chain fatty acids and their esters, alcohols, amino acids, and small peptides, all of which are known to contribute significantly to cheese flavor (Seitz, 1990; Urbach, 1993; Singh et al., 2003). This extraction procedure significantly reduced interfering compounds and improved spectral definition in the region 1,800 to $900 \mathrm{~cm}^{-1}$ that contains signal from several flavor-related compounds.

\section{Classification of Cheddar Cheese Based on Flavor}

Direct examination of Cheddar cheese had low discrimination and inconsistent spectra presumably because of the presence of interfering compounds and 


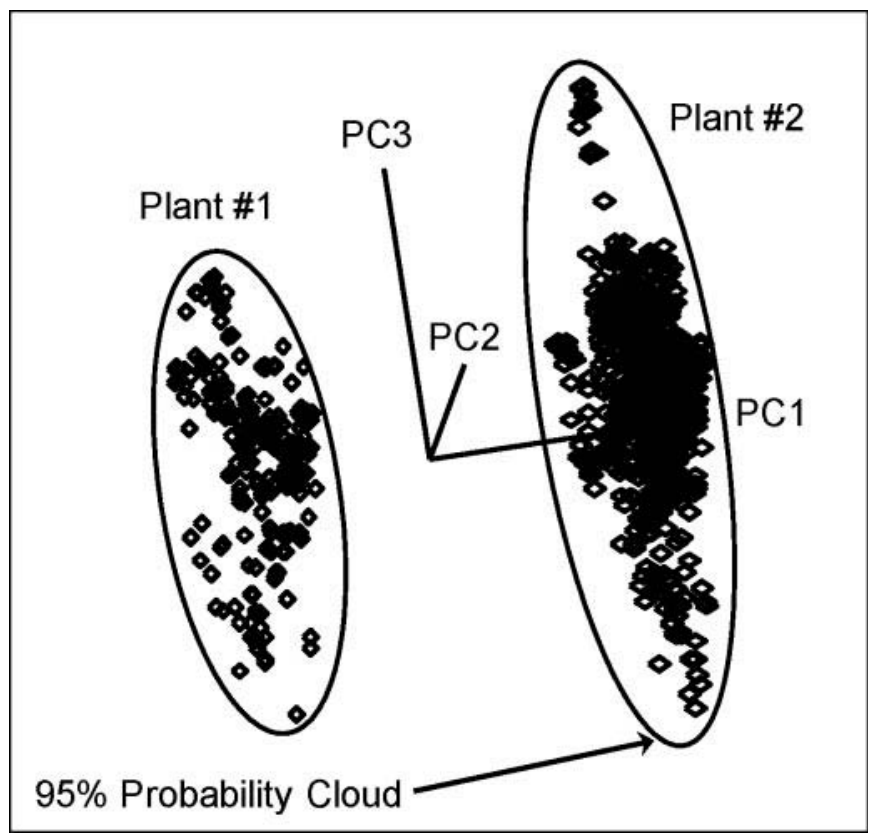

Figure 2. Soft independent modeling of class anology (SIMCA) classification plot of samples from the 2 production plants. The midinfrared spectra were transformed into their second derivative, meancentered and normalized prior multivariate analysis. The samples clustered distinctly indicating marked difference in the chemical composition of samples from the 2 production plants. The $95 \%$ probability cloud indicates the probability of a sample belonging to the cluster in which it is located.

heterogeneity in cheese samples (data not shown). Visual comparison of the raw spectra of Cheddar cheese water-soluble fractions showed numerous differences between cheeses in the spectral regions 1,800 to $900 \mathrm{~cm}^{-1}$. Using the derivatized spectra, classification models based on SIMCA were developed to discriminate cheese from the 2 production plants. The SIMCA models were developed by computing a small number of orthogonal variables (PC) that explained as much of the variation as possible between the samples, while preserving the relevant information and eliminating random noise (Mark, 2001). The SIMCA classification plot, a projection of the original data onto first $3 \mathrm{PC}$, allowed the visualization of well-separated clustering among the samples, whose orientation in 3D space correlated with their flavor quality. The SIMCA also provided a $95 \%$ probability cloud, which means that there is $95 \%$ probability that the samples within that cloud belong to the same flavor category.

Many factors such as ingredients, milk composition, and bacterial starter culture used for fermentation influence the final flavor of Cheddar cheese (Chen et al., 1998; Bachmann et al., 1999). Furthermore, many cheese plants develop their own strains of nonstarter lactic acid bacteria with time, which significantly in-

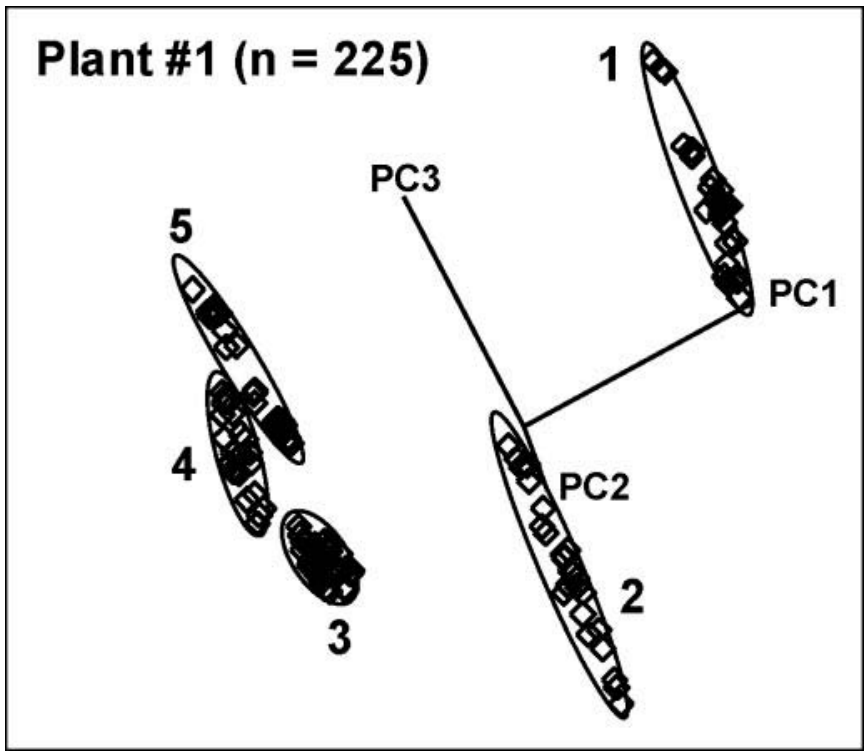

Figure 3. Soft independent modeling of class analogy (SIMCA) classification plot of samples from plant 1 . The samples were projected against the first 3 principal components $(\mathrm{PC})$ that explained the largest amount of variance among the samples. All 5 samples formed distinct clusters in 3-dimensional space based on their flavor quality descriptors $(1=$ fermented; $2=$ unclean; $3=$ slight sour; $4=\operatorname{good}$ Cheddar; and $5=$ slight burnt).

fluence the final composition and flavor of the cheese. Our research showed marked differences in the chemical composition of samples from the 2 production plants (Figure 2). This, aside from indicating the possibility of identifying the production plant based on the spectra, suggests that a separate flavor classification model is needed for each production plant. The discrimination of the 5 samples from plant 1 is shown in Figure 3; all 5 samples formed tight and distinct clusters. The distance between the clusters in a SIMCA plot is represented by the interclass distance (ICD). The greater the distance between 2 clusters, the greater is the difference in composition of samples belonging to those clusters. As a rule of thumb, a distance of $>3$ indicates that the samples are well separated and hence different (Kvalheim and Karstand, 1992). Table 1 shows the ICD values of the 5 samples from plant 1 . The samples with fermented flavor and unclean flavor, which are considered undesirable, clustered far from other samples. The ICD of the fermented sample and unclean sample from good Cheddar were 28.21 and 13.96 units, respectively. Samples with slight burn and slight sour notes, which were not major flavor defects, clustered relatively close to the good Cheddar, with ICD of 6.14 and 3.70, respectively.

Samples from plant 2 also exhibited very good clustering based on their flavor quality (Figure 4). The ICD for samples from plant 2 are shown in Table 2. Samples 
Table 1. Interclass distances of Cheddar cheese samples from plant $1^{1}$

\begin{tabular}{lrrrrr}
\hline Sample $^{2}$ & 1 & \multicolumn{1}{c}{2} & \multicolumn{1}{c}{3} & \multicolumn{1}{c}{5} \\
\hline 1 & 0.00 & 4.75 & 6.14 & 29.62 & 12.49 \\
2 & 4.75 & 0.00 & 3.70 & 31.56 & 13.99 \\
3 & 6.14 & 3.70 & 0.00 & 28.21 & 13.96 \\
4 & 29.62 & 31.56 & 28.21 & 0.00 & 15.85 \\
5 & 12.49 & 13.99 & 13.96 & 15.85 & 0.00 \\
\hline
\end{tabular}

${ }^{1}$ The greater the distance between 2 samples, the greater the difference between them.

${ }^{2}$ Sample 1 = slight burn; sample 2 = sour; sample 3 = good; sample 4 $=$ fermented; and sample $5=$ unclean.

with fermented flavor and low flavor clustered far from good Cheddar (ICD >3.0). Their ICD from good Cheddar were 5.08 and 8.48, respectively. Samples with minor defects such as slight sour, slight acid, sulfide flavor, and slight low flavor clustered close to the good Cheddar, with relatively low ICD. In flavor categories that contained more than one sample (good Cheddar, and sour and slight acid), samples within the flavor category clustered close to each other (ICD <3.0) and away from other categories (ICD >3.0). All the samples in the sour and slight acid categories (samples 8, 9, 10 , and 11) had ICD $<3.0$ among themselves and $>3.0$ for samples outside the cluster (Table 2). Similarly, the good Cheddar samples (samples 6 and 14) had ICD of $<3.0$ between themselves and ICD $>3.0$ from other clusters. These data clearly indicate that the developed extraction method combined with the FT-IR technique has the potential for rapid quality control of Cheddar cheese flavor.

\section{Identification of Infrared Bands Responsible for Classification}

The spectral wavenumbers and the associated chemical functional groups that were responsible for the

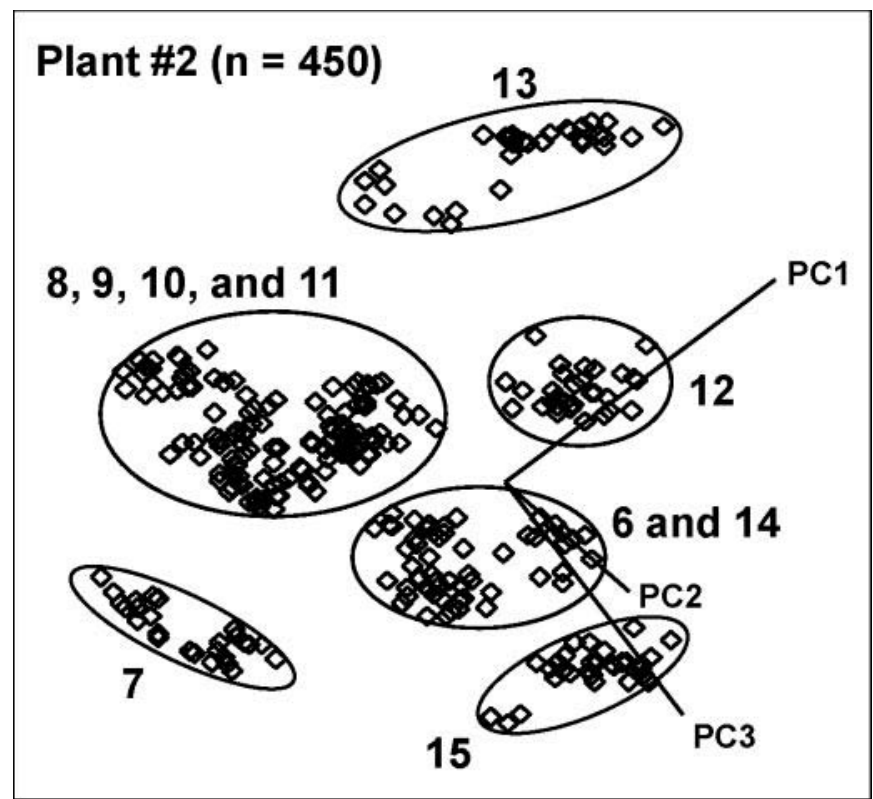

Figure 4. Soft independent modeling of class analogy (SIMCA) classification plot of samples from plant 2. The orientation of the clusters in the SIMCA plot correlated with the flavor of the cheese samples. Samples 6 and $14=$ good Cheddar; sample $7=$ fermented; samples $8,9,10$, and $11=$ sour and slight acid; sample $12=$ slight low flavor and slight sour; sample $13=$ low flavor; and sample $15=$ sulfide. $\mathrm{PC}=$ principal components.

classification of the cheeses in the SIMCA plot can be identified using the discriminating power plot. In the discriminating power plot each wavenumber in the spectral range is plotted against its importance in discriminating the samples that are in the model. The greater the value of discriminating power, the greater the role of that wavenumber in classifying the samples. In other words, the greater the discriminating power, the greater the difference between the samples in compounds or functional groups associated with that wavenumber. The spectral regions and the associated functional groups and compounds responsible

Table 2. Interclass distances of Cheddar cheese samples from plant $2^{1}$

\begin{tabular}{|c|c|c|c|c|c|c|c|c|c|c|}
\hline Sample $^{2}$ & 6 & 7 & 8 & 9 & 10 & 11 & 12 & 13 & 14 & 15 \\
\hline 7 & 5.08 & 0.00 & 6.02 & 7.70 & 7.06 & 7.18 & 9.79 & 12.56 & 4.63 & 7.49 \\
\hline 8 & 3.81 & 6.02 & 0.00 & 2.66 & 1.96 & 1.39 & 5.62 & 8.71 & 3.58 & 8.89 \\
\hline 10 & 5.42 & 7.06 & 1.96 & 2.23 & 0.00 & 1.80 & 6.35 & 8.73 & 3.62 & 10.29 \\
\hline 11 & 3.97 & 7.18 & 1.39 & 2.79 & 1.80 & 0.00 & 5.17 & 8.74 & 3.25 & 7.94 \\
\hline 12 & 5.39 & 9.79 & 5.62 & 6.06 & 6.35 & 5.17 & 0.00 & 5.61 & 6.29 & 6.49 \\
\hline 15 & 5.51 & 7.49 & 8.89 & 10.37 & 10.29 & 7.94 & 6.49 & 12.07 & 7.82 & 0.00 \\
\hline
\end{tabular}

${ }^{1}$ The greater the distance between 2 samples, the greater the difference between them.

${ }^{2}$ Samples 6 and $14=$ good Cheddar; sample $7=$ fermented; samples 8, 9, 10, and $11=$ sour and slight acid; sample $12=$ slight low flavor and slight sour; sample $13=$ low flavor; and sample $15=$ sulfide. 


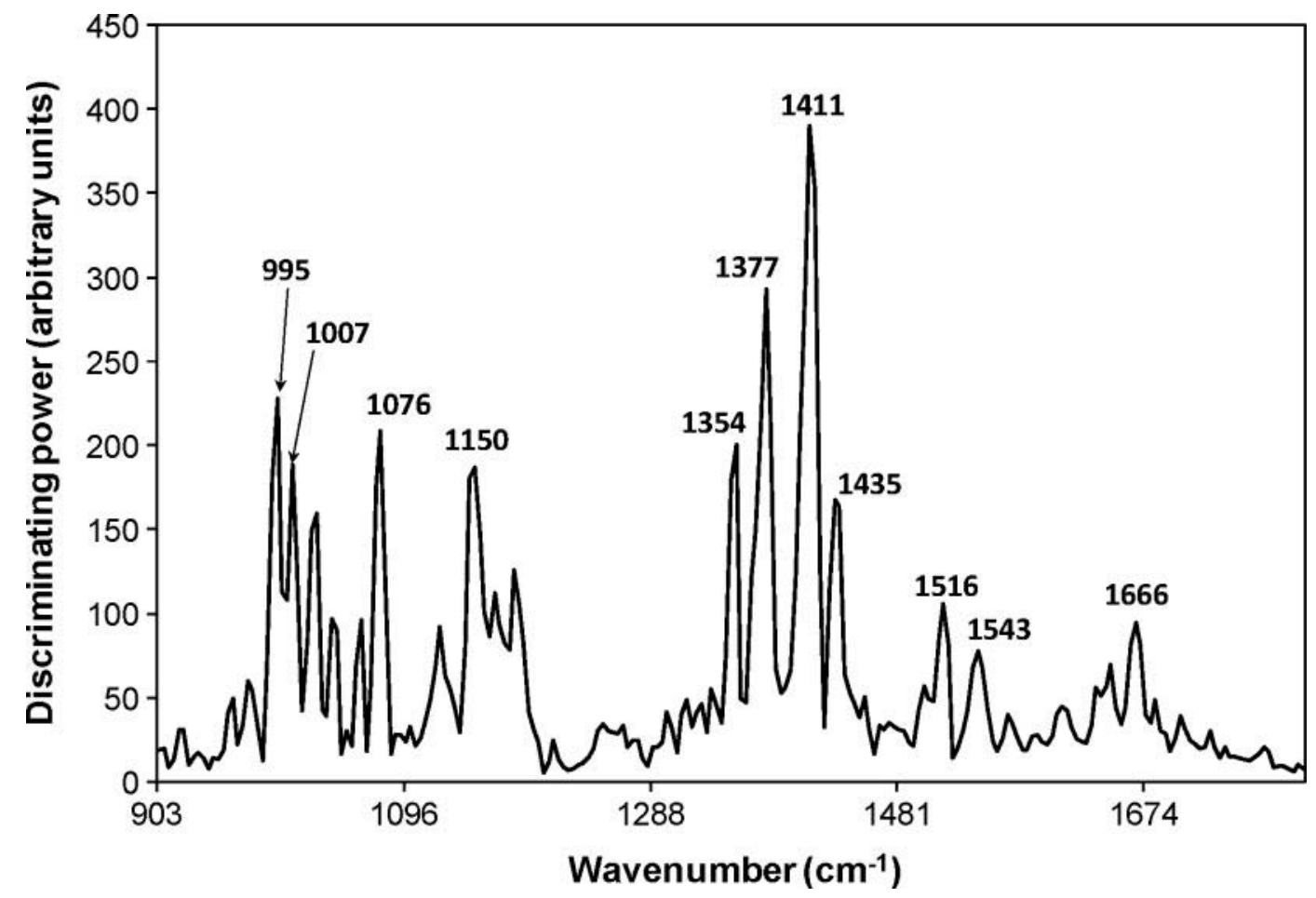

Figure 5. Discriminating power plot for classification of Cheddar cheese samples. The regions of the Fourier transform infrared spectra that contribute to the discrimination of the cheese samples based on their flavor are highlighted. The greater the discriminating power at a particular wavenumber, the greater the difference between the samples in the functional group associated with that wavenumber.

for the differentiation of the Cheddar cheese samples are highlighted in Figure 5. The spectral range 1,800 to $900 \mathrm{~cm}^{-1}$ was found to be important in the analysis of cheese flavor by FT-IR. This region contained several wavenumbers that played a significant role in discriminating the samples (indicated by their high discriminating power values). The major bands responsible for the classification were $1,411,1,377$, and 1,354 $\mathrm{cm}^{-1}$. These bands mainly include stretching vibrations of carboxyl groups, C-H bending vibrations of methyl groups, and C-N stretching vibrations of amines (Guillen and Cabo, 1997; Coates, 2000). Koca et al. (2007) associated $1,412 \mathrm{~cm}^{-1}$ with $\mathrm{C}-\mathrm{H}$ symmetric bending vibrations of short- and medium-chain fatty acids. Some organic sulfates have also been reported to absorb in this region (Coates, 2000). Stretching vibrations of C-O groups appear in the region from 1,200 to $1,000 \mathrm{~cm}^{-1}$ (Rodriguez-Saona et al., 2006). These signals could be attributed to $\mathrm{C}-\mathrm{O}$ containing compounds including alcohols, organic acids, fatty acids, lactones, and keto acids. Carboxyl groups of acids also absorb at around $1,435 \mathrm{~cm}^{-1}$. Several other bands $-995 \mathrm{~cm}^{-1}$ associated with $\mathrm{C}-\mathrm{H}$ in-plane bends of aromatic compounds, 1,543 and $1,516 \mathrm{~cm}^{-1}$ associated with aromatic nitrogen compounds, and $1,666 \mathrm{~cm}^{-1}$ associated with amide I bands of peptides - also influenced the discrimination of Cheddar cheese based on flavor.

\section{CONCLUSIONS}

A rapid, simple, and reliable sample preparation method and FT-IR technique was developed for analysis of Cheddar cheese flavor quality. The infrared spectra could be correlated to specific flavor notes such as fermented, sour, and unclean, and differentiated using multivariate classification models. The total analysis time, including sample preparation time, was less than 20 min per sample. The results indicate that this technique could be used for detection of flavor quality defects in Cheddar cheese. Furthermore, differences in the composition of cheese from different production plants can be elucidated. The developed technique shows great promise as a rapid and simple tool for application to cheese analysis and can save time and money for the cheese industry. It will enable better quality control and rapid monitoring of ripening process to achieve cheese of desired flavor quality. Additionally, this method will initiate and accelerate further studies on rapid compositional analysis and cheese ripening process using FT-IR spectroscopy. 


\section{ACKNOWLEDGMENT}

The project was supported by the USDA Cooperative State Research, Education and Extension Service, special research grant number 2006-34328-17149.

\section{REFERENCES}

Adda, J., J. C. Gripon, and L. Vassal. 1982. The chemistry of flavor and texture generation in cheese. Food Chem. 9:115-129.

Akalin, A. S., S. Gonc, and Y. Akba. 2002. Variation in organic acids content during ripening of pickled white cheese. J. Dairy Sci. 85:1670-1676.

Alli, I., M. Okoniewska, B. F. Gibbs, and Y. Konishi. 1998. Identification of peptides in Cheddar cheese by electrospray ionization mass spectrometry. Int. Dairy J. 8:643-649.

Aston, J., and L. K. Creamer. 1986. Contribution of the components of water-soluble fraction to the flavor Cheddar cheese. N. Z. Dairy Sci. Technol. 21:229-248.

Bachmann, H. P., U. Butikofer, and J. Meyer. 1999. Prediction of flavor and texture development in Swiss-type cheeses. Lebensm. Wiss. Technol. 32:284-289.

Cattaneo, T. M. P., C. Giardina, N. Sinelli, M. Riva, and R. Giangiacomo. 2005. Application of FT-NIR and FT-IR spectroscopy to study the shelf-life of Crescenza cheese. Int. Dairy J. 15:693-700.

Chen, M., and J. Irudayaraj. 1998. Sampling technique for cheese analysis by FTIR spectroscopy. J. Food Sci. 63:96-99.

Chen, M., J. Irudayaraj, and D. J. McMahon. 1998. Examination of full fat and reduced fat Cheddar cheese during ripening by Fourier transform infrared spectroscopy. J. Dairy Sci. 81:2791-2797.

Coates, J. 2000. Interpretation of infrared spectra: A practical approach Pages 10815-10837 in Encyclopedia of Analytical Chemistry. R. R. Meyers, ed. John Wiley and Sons Ltd., Chichester, UK.

Engel, E., J. B. Lombardot, A. Garem, N. Leconte, C. Septier, J. L. Le Quere, and C. Salles. 2002. Fractionation of the water-soluble extract of cheese made from goat's milk by filtration methods: Behavior of fat and volatile compounds. Int. Dairy J. 12:609619.

Engels, W. J. M., R. Dekker, C. de Jong, R. Neeter, and S. Visser. 1997. A comparative study of volatiles compounds in the watersoluble fraction of various types of ripened cheese. Int. Dairy J. $7: 255-263$.

Farkye, N. Y., and P. F. Fox. 1990. Objective indices of cheese ripening. Trends Food Sci. Technol. 1:37-40.

Guillen, M. D., and N. Cabo. 1997. Infrared spectroscopy in the study of edible oils and fats. J. Sci. Food Agric. 75:1-11.

Kansiz, M., P. Heraud, B. Wood, F. Burden, J. Beardall, and D. McNaughton. 1999. Fourier transform infrared microspectroscopy and chemometrics as a tool for the discrimination of cyanobacterial strains. Phytochemistry 52:407-417.

Karoui, R., E. Dufour, L. Pillonel, D. Picque, T. Cattenoz, and J. O. Bosset. 2004. Determining the geographic origin of Emmental cheeses produced during winter and summer using a technique based on concatenation of MIR and fluorescence spectroscopic data. Eur. Food Res. Technol. 219:184-189.

Koca, N. L. E. Rodriguez-Saona, W. J. Harper, and V. B. Alvarez. 2007. Application of Fourier transform infrared (FTIR) spectroscopy for monitoring short-chain free fatty acids in Swiss cheese. J. Dairy Sci. 90:3596-3603.

Kosikowski, F. V., and G. Mocquot. 1958. Advances in cheese technology. FAO Agric. Stud. No. 38. Food and Agriculture Organization (FAO), Rome, Italy.

Kvalheim, O. M., and T. V. Karstand. 1992. SIMCA —Classification by means of disjoint cross validated principal components models. Pages 209-248 in Multivariate Pattern Recognition in Chemometrics: Illustrated by Case Studies. R. G. Brereton, ed. Elsevier, Amsterdam, the Netherlands.

Lues, J. F. R., and A. C. M. Bekker. 2002. Mathematical expression for organic acids in early ripening of a Cheddar cheese. J. Food Compost. Anal. 15:11-17.

Mark, H. 2001. Multilinear regression and principal component analysis. Pages 129-184 in Handbook of Near-Infrared Analysis. D. A. Burns, and E. W. Ciurczak, ed. Taylor and Francis, New York, NY.

McQueen, D. H., R. Wilson, A. Kinnunen, and E. P. Jensen. 1995 Comparison of two infrared spectroscopic methods for cheese analysis. Talanta 42:2007-2015.

McSweeney, P. L. H., and M. J. Sousa. 2000. Biochemical pathways for the production of flavour compounds in cheeses during ripening: A review. Lait 80:293-324.

National Agricultural Statistics Service (NASS). 2007. United States Department of Agriculture, http://www.nass.usda.gov/, Accessed Mar. 31, 2007.

Partidario, A. M., M. Barbosa, and L. V. Boas. 1998. Free fatty acids, triglycerides and volatile compounds in Serra da Estrela cheesechanges throughout ripening. Int. Dairy J. 8:873-881.

Rodriguez-Saona, L. E., N. Koca, W. J. Harper, and V. B. Alvarez. 2006. Rapid determination of Swiss cheese composition by Fourier transform infrared/attenuated total reflectance spectroscopy. J. Dairy Sci. 89:1407-1412.

Seitz, E. W. 1990. Microbial- and enzyme-induced flavor in dairy foods. J. Dairy Sci. 73:3664-3691.

Singh, T. K., M. A. Drake, and K. R. Cadwallader. 2003. Flavor of Cheddar cheese: A chemical and sensory perspective. Comp. Rev. Food Sci. Food Saf. 2:166-189.

Subramanian, A., and L. E. Rodriguez-Saona, inventors. 2008. Rapid extraction method for analysis of cheese flavor using infrared spectroscopy. The Ohio State University, assignee. Provisional Appl. Ser. No. 61,890.

Thierry, A., M. B. Maillard, and J. L. Le Quere. 1999. Dynamic headspace analysis of Emmental aqueous phase as a method to quantify changes in volatile flavor compounds during ripening. Int. Dairy J. 9:453-463.

Urbach, G. 1993. Relations between cheese flavor and chemical composition. Int. Dairy J. 3:389-422. 